\title{
Boundary Layer Flow Past a Wedge Moving in a Nanofluid
}

\author{
Waqar A. Khan ${ }^{1}$ and I. Pop ${ }^{2}$ \\ ${ }^{1}$ Department of Engineering Sciences, PN Engineering College, National University of Sciences and Technology, \\ Karachi 75350, Pakistan \\ ${ }^{2}$ Faculty of Mathematics, University of Cluj, CP 253, 3400 Cluj, Romania
}

Correspondence should be addressed to Waqar A. Khan; wkhan_2000@yahoo.com

Received 30 March 2013; Revised 16 April 2013; Accepted 19 April 2013

Academic Editor: Oluwole Daniel Makinde

Copyright (C) 2013 W. A. Khan and I. Pop. This is an open access article distributed under the Creative Commons Attribution License, which permits unrestricted use, distribution, and reproduction in any medium, provided the original work is properly cited.

\begin{abstract}
The problem of steady boundary layer flow past a stretching wedge with the velocity $u_{w}(x)$ in a nanofluid and with a parallel free stream velocity $u_{e}(x)$ is numerically studied. It is assumed that at the stretching surface the temperature $T$ and the nanoparticle fraction $C$ take the constant values $T_{w}$ and $C_{w}$, respectively. The ambient values (inviscid fluid) of $T$ and $C$ are denoted by $T_{\infty}$ and $C_{\infty}$, respectively. The boundary layer governing partial differential equations of mass, momentum, thermal energy, and nanoparticles recently proposed by Kuznetsov and Nield $(2006,2009)$, are reduced to ordinary differential equations along with the corresponding boundary conditions. These equations are solved numerically using an implicit finite-difference method for some values of the governing parameters, such as $\beta, \lambda, \operatorname{Pr}, \mathrm{Le}, N_{b}$, and $N_{t}$, which are the measure of the pressure gradient, moving parameter, Prandtl number, Lewis number, the Brownian motion parameter, and the thermophoresis parameter, respectively.
\end{abstract}

\section{Introduction}

Historically, the steady laminar flow past a fixed wedge was first analyzed by Falkner and Skan [1] to illustrate the application of Prandtl's boundary layer theory. With a similarity transformation, the boundary layer equations are reduced to an ordinary differential equation, which is well known as the Falkner-Skan equation. This equation includes nonuniform flow, that is, outer flows which, when evaluated at the wall, takes the form $a x^{m}$, where $x$ is the coordinate measured along the wedge wall and $a(>0)$, and $m$ are constants. There is a large body of literature on the solutions of FalknerSkan equation, see Hartree [2], Stewartson [3], Chen and Libby [4], Rajagopal et al. [5], Botta et al. [6], Brodie and Banks [7], Heeg et al. [8], Zaturska and Banks [9], Kuo [10], Pantokratoras [11], and so forth. Liao [12] has developed an analytical technique, named homotopy analysis method (HAM), and presented a uniformly valid analytic solution of Falkner-Skan equation for the wedge parameter $\beta$ in the range $-0.19884 \leq \beta \leq 2$. This solution has been extended by Abbasbandy and Hayat [13] by including the magnetic effects. Also, there are some very recently published papers on this problem by Liu and Chang [14], Fang and Zhang [15], and Pal and Mondal [16]. A very good list of references on this problem can be found in the recent papers by Harris et al. [17-19]. The flows predicted by the Falkner-Skan solutions are naturally assumed to be described adequately by the boundary layer equations which are parabolic in character. However, the use of the similarity method of solution cannot take account of the "initial" condition in general and so the resulting solutions are assumed to be valid, if at all, in some asymptotic sense (see Banks [20]). This is the case for the Falkner-Skan flows that has been shown rigorously by Serrin [21] for $0 \leq \beta \leq 2$. However, all these papers are for the Falkner-Skan boundary layer flow over a fixed wedge placed in a moving fluid. In a very interesting paper, Riley and Weidman [22] and Ishak et al. [23] have studied multiple solutions of the Falkner-Skan equation for flow past a stretching boundary when the external velocity and the boundary velocity are each proportional to the same power law of the downstream distance. Boundary layer behavior over a moving continuous solid surface is an important type of flow occurring in several engineering processes. For example, the thermal processing of sheet-like materials is 
a necessary operation in the production of paper, linoleum, polymeric sheets, wire drawing, drawing of plastic films, metal spinning, roofing shingles, insulating materials, and fine-fiber Matts.

The aim of the present paper is to extend the papers by Riley and Weidman [22] and Ishak et al. [23] to the case when the wedge moves in a nanofluid. Enhancement of heat transfer is essential in improving performances and compactness of electronic devices. Usual cooling agents (water, oil, etc.) have relatively small thermal conductivities, and therefore heat transfer is not very efficient. Thus, to augment thermal characteristics very small size particles (nanoparticles) were added to fluids forming the so-called nanofluids. These suspensions of nanoparticles in fluids have physical and chemical properties depending on the concentration and the shape of particles. It was discovered that a small fraction of nanoparticles added in a base fluid leads to a large increase of the fluid thermal conductivity. The chaotic movement of the nanoparticles and sleeping between the fine particles and fluid generates the thermal dispersion effect, and this leads to an increase in the energy exchange rates in fluid. Based on the fact that for small size suspended particles (smaller than $100 \mathrm{~nm}$ ) nanofluids behave more like a fluid than a fluidsolid mixture. Xuan and Roetzel [24] proposed a thermal dispersion model for a single-phase nanofluid. Kuznetsov and Nield [25] extended the classical boundary layer analysis of forced convection over a wedge with an attached porous substrate. In another two papers, Nield and Kuznetsov [26-28] extended the classical Cheng and Minkowycz [29] problem of boundary layer flow over a vertical flat plate in a porous medium saturated by a nanofluid and also the problem of double-diffusive natural convective boundary layer flow in a porous medium saturated by a nanofluid, presenting similarity solutions. Kuznetsov and Nield [30] extended also the Pohlhausen-Kuiken-Bejan problem to the case of a binary nanofluid using Buongiorno model and presented a similarity solution. Also, there are several very good recently published papers on nanofluids, for example, papers by Narayana and Sibanda [31] and Kameswaran et al. [32, 33]. However, the broad range of current and future applications of nanofluids is discussed in the review article by Woong and Leon [34], which includes automotive, electronics, biomedical, and heat transfer applications, besides other applications such as nanofluid detergent. In a recent review article by Saidur et al. [35], the authors also presented some applications of nanofluids in industrial, commercial, residential, and transportation sectors based on the available literatures. Recent critical reviews of the state-of-the-art of nanofluids research for heat transfer application were conducted by Mahian et al. [36] and Behar et al. [37].

In the present paper, the thermal dispersion model is similar with that proposed by Nield and Kuznetsov [28] and Kuznetsov and Nield [30]. The mentioned literature survey indicates that there is no study on the boundary layer flow past a wedge in a nanofluid. It is worth mentioning to this end that nanotechnology has been widely used in the industry since materials with sizes of nanometers possess unique physical and chemical properties.

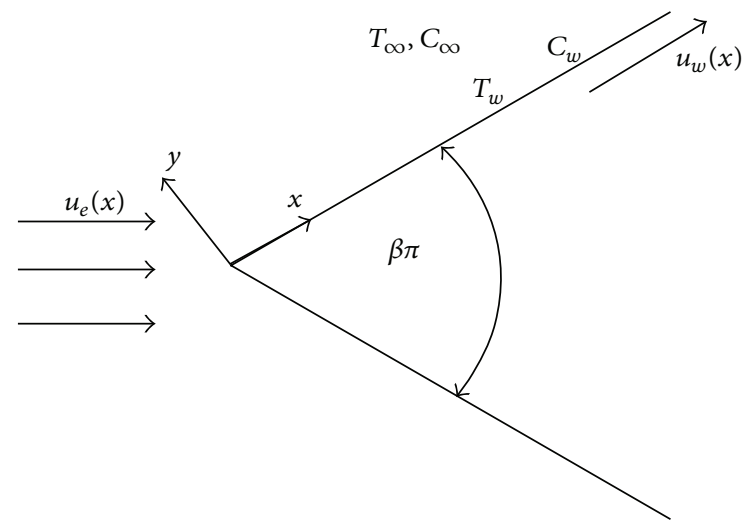

FIGURE 1: Schematic diagram of a stretching wedge.

\section{Problem Formulation and Basic Equations}

We consider the boundary layer flow past an impermeable stretching wedge moving with the velocity $u_{w}(x)$ in a nanofluid, and the free stream velocity is $u_{e}(x)$, where $x$ is the coordinate measured along the surface of the wedge as shown in Figure 1. It should be noted that the case of $u_{w}(x)>0$ corresponds to a stretching wedge surface and $u_{w}(x)<0$ corresponds to a contracting wedge surface, respectively. It is assumed that at the stretching surface, the temperature $T$ and the nanoparticle fraction $C$ take constant values $T_{w}$ and $C_{w}$, respectively. The ambient values, attained as $y$ tends to infinity, of $T$ and $C$ are denoted by $T_{\infty}$ and $C_{\infty}$, respectively. Under these assumptions, it can be shown that the steady boundary layer equations of mass, momentum, thermal energy, and nanoparticles for nanofluids can be written in Cartesian coordinates $x$ and $y$ as, see Nield and Kuznetsov [28], and Kuznetsov and Nield [30],

$$
\begin{gathered}
\frac{\partial u}{\partial x}+\frac{\partial v}{\partial y}=0 \\
u \frac{\partial u}{\partial x}+v \frac{\partial u}{\partial y}=u_{e} \frac{d u_{e}}{d x}+v \frac{\partial^{2} u}{\partial y^{2}} \\
u \frac{\partial T}{\partial x}+v \frac{\partial T}{\partial y} \\
=\alpha \frac{\partial^{2} T}{\partial y^{2}}+\tau\left[D_{B} \frac{\partial C}{\partial y} \frac{\partial T}{\partial y}+\left(\frac{D_{T}}{T_{\infty}}\right)\left(\frac{\partial T}{\partial y}\right)^{2}\right], \\
u \frac{\partial C}{\partial x}+v \frac{\partial C}{\partial y}=D_{B} \frac{\partial^{2} C}{\partial y^{2}}+\left(\frac{D_{T}}{T_{\infty}}\right) \frac{\partial^{2} T}{\partial y^{2}},
\end{gathered}
$$

subject to the boundary conditions

$$
\begin{array}{r}
v=0, \quad u=u_{w}(x)=-\lambda u_{e}(x), \quad T=T_{w}, \quad C=C_{w} \\
\text { at } y=0, \\
u=u_{e}(x), \quad T=T_{\infty}, \quad C=C_{\infty} \quad \text { as } y \longrightarrow \infty .
\end{array}
$$

Here $u$ and $v$ are velocity components along the axes $x$ and $y$, respectively, $\alpha$ is the thermal diffusivity, $v$ is the kinematic 
viscosity, $D_{B}$ is the Brownian diffusion coefficient, $D_{T}$ is the thermophoretic diffusion coefficient and $\tau=(\rho c)_{p} /(\rho c)_{f}$ with $\rho$ being the density, $c$ is volumetric volume expansion coefficient, and $\rho_{p}$ is the density of the particles.

In order to get similarity solutions of (1)-(5), we assume that $u_{w}(x)$ and $u_{e}(x)$ have the following form:

$$
u_{w}(x)=a x^{m}, \quad u_{e}(x)=c x^{m},
$$

where $a, c$, and $m(0 \leq m \leq 1)$ are positive constants. Therefore, the constant moving parameter $\lambda$ in (6) is defined as $\lambda=c / a$, so that $\lambda<0$ corresponds to a stretching wedge, $\lambda>0$ corresponds to a contracting wedge, and $\lambda=0$ corresponds to a fixed wedge, respectively. Thus, we look for a similarity solution of (1)-(4) with the boundary conditions (5) of the following form:

$$
\begin{gathered}
\psi=\left(\frac{2 u_{e} x v}{1+m}\right)^{1 / 2} f(\eta), \quad \theta(\eta)=\frac{T-T_{\infty}}{T_{w}-T_{\infty}}, \\
\phi(\eta)=\frac{C-C_{\infty}}{C_{w}-C_{\infty}}, \\
\eta=\left(\frac{(1+m) u_{e}}{2 x v}\right)^{1 / 2} y,
\end{gathered}
$$

where the stream function $\psi$ is defined in the usual way as $u=\partial \psi / \partial y$ and $v=-\partial \psi / \partial x$. On substituting (7) into (2)-(4), we obtain the following ordinary differential equations:

$$
\begin{gathered}
f^{\prime \prime \prime}+f f^{\prime \prime}+\beta\left(1-f^{\prime 2}\right)=0, \\
\frac{1}{\operatorname{Pr}} \theta^{\prime \prime}+f \theta^{\prime}+N_{b} \phi^{\prime} \theta^{\prime}+N_{t} \theta^{\prime 2}=0, \\
\phi^{\prime \prime}+\operatorname{Le} f \phi^{\prime}+\frac{N_{t}}{N_{b}} \theta^{\prime \prime}=0,
\end{gathered}
$$

subject to the boundary conditions

$$
\begin{gathered}
f(0)=0, \quad f^{\prime}(0)=-\lambda, \quad \theta(0)=1, \quad \phi(0)=1, \\
f^{\prime}(\infty)=1, \quad \theta(\infty)=0, \quad \phi(\infty)=0,
\end{gathered}
$$

where primes denote differentiation with respect to $\eta$, and the five parameters are defined by

$$
\begin{gathered}
\beta=\frac{2 m}{1+m}, \quad \operatorname{Pr}=\frac{\nu}{\alpha}, \\
\mathrm{Le}=\frac{v}{D_{B}}, \quad N_{b}=\frac{(\rho c)_{p} D_{B}\left(\phi_{w}-\phi_{\infty}\right)}{(\rho c)_{f} v}, \\
N_{t}=\frac{(\rho c)_{p} D_{T}\left(T_{w}-T_{\infty}\right)}{(\rho c)_{f} T_{\infty} v} .
\end{gathered}
$$

Here $\beta$, Pr, Le, $N_{b}$, and $N_{t}$ are the measure of the pressure gradient, Prandtl number, Lewis number, the Brownian motion, and the thermophoresis parameters, respectively. It is important to note that this boundary value problem reduces to the classical Falkner-Skan's [1] problem of the boundary layer flow of a viscous and incompressible fluid past a fixed wedge, when $\lambda, N_{b}$, and $N_{t}$ are all zero in (9) and (10).
TABLE 1: Comparison of the values of $f^{\prime \prime}(0)$ for several values of $m$ when $\lambda=0$.

\begin{tabular}{lcccc}
\hline$m$ & Yih [38] & Yacob et al. [39] & White [40] & Present results \\
\hline 0 & 0.4696 & 0.4696 & 0.4696 & 0.4696 \\
$1 / 11$ & 0.6550 & 0.6550 & 0.6550 & 0.6550 \\
$1 / 5$ & 0.8021 & 0.8021 & 0.8021 & 0.8021 \\
$1 / 3$ & 0.9276 & 0.9276 & 0.9277 & 0.9277 \\
$1 / 2$ & - & - & 1.0389 & 1.0389 \\
1 & & 1.2326 & 1.2326 & 1.2326 \\
\hline
\end{tabular}

TABLE 2: Comparison of the values of $-\theta^{\prime}(0)$ for various values of $m$ when $\lambda=N_{b}=N_{t}=0$.

\begin{tabular}{lccc}
\hline$m$ & Kuo [41] & Blasius [42] & Present \\
\hline 0 & 0.8673 & 0.8673 & 0.8769 \\
1.0 & 1.1147 & 1.1152 & 1.1279 \\
\hline
\end{tabular}

2.1. Numerical Method. Equations (8)-(10) with boundary conditions (11) are solved numerically using an implicit finite-difference scheme known as the Keller-box method, as described by Cebeci and Bradshaw [43, 44]. In this method, (8)-(10) are reduced to first-order equations, and using central differences, the algebraic equations are obtained. These equations are then linearized by Newton's method. The linear equations are then solved using block-tridiagonal elimination technique. The boundary conditions for $\eta \rightarrow \infty$ are replaced by $f^{\prime}\left(\eta_{\max }\right)=1, \theta\left(\eta_{\max }\right)=0, \phi\left(\eta_{\max }\right)=0$, where $\eta_{\max }=12$. The step size is taken as $\Delta \eta=0.001$, and the convergence criteria is set to $10^{-6}$.

\section{Results and Discussion}

Table 1 shows the comparison of the values of $f^{\prime \prime}(0)$ for several values of $m$ when $\lambda=0$ with those reported by [38$40]$. On the other hand, Table 2 compares the values of $-\theta^{\prime}(0)$ obtained from (8) and (9) for $m=0$ and several values of Pr when $\lambda=N_{b}=N_{t}=0$ with those of [41, 42]. It is seen from these tables that the results are in very good agreement. Therefore, we are deeply confident that the present numerical results are correct and very accurate.

The effect of the wedge parameter $m$ on the dimensionless velocity is shown in Figure 2(a) for stretching wedge and in Figure 2(b) for shrinking wedge. It is observed that the dimensionless velocity at the surface increases/decreases with stretching/shrinking parameters. This is just due to increase/decrease in the stretching/shrinking velocities. In both cases, the hydrodynamic boundary layer thickness decreases with increasing wedge parameter $m$. The effects of Prandtl numbers on the dimensionless temperature are shown in Figures 3(a) and 3(b) for shrinking and stretching wedge, respectively. The Prandtl number is defined as the ratio of momentum diffusivity to thermal diffusivity. When $\operatorname{Pr}=1$, both momentum and thermal diffusivities are comparable, but when $\operatorname{Pr}>1$, the momentum diffusivity is greater than thermal diffusivity and the thermal boundary layer thickness decreases with increasing Prandtl number. 


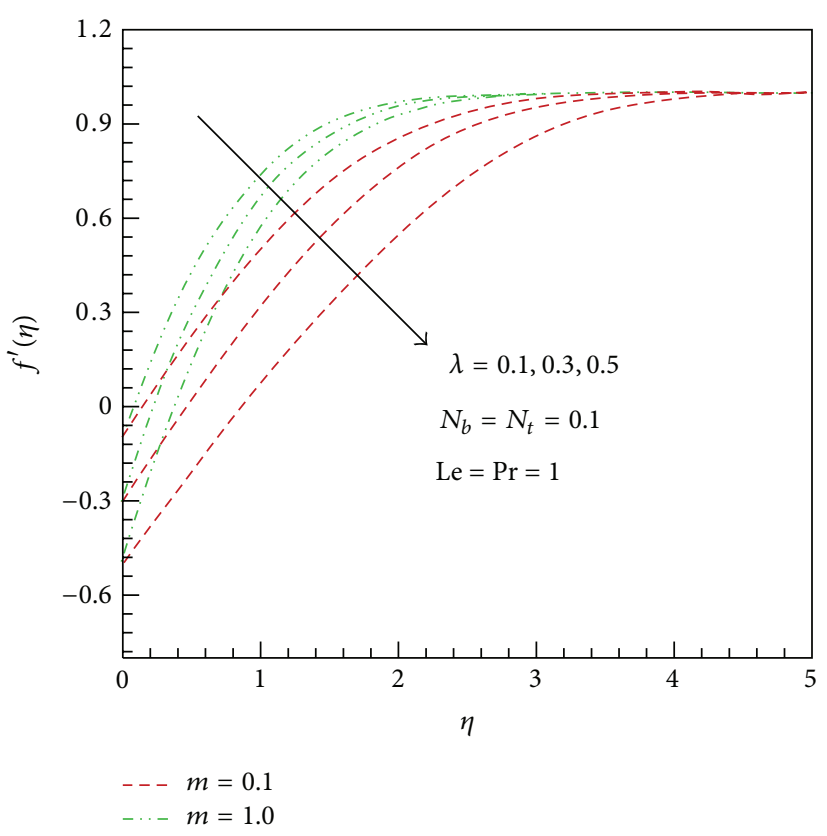

(a)

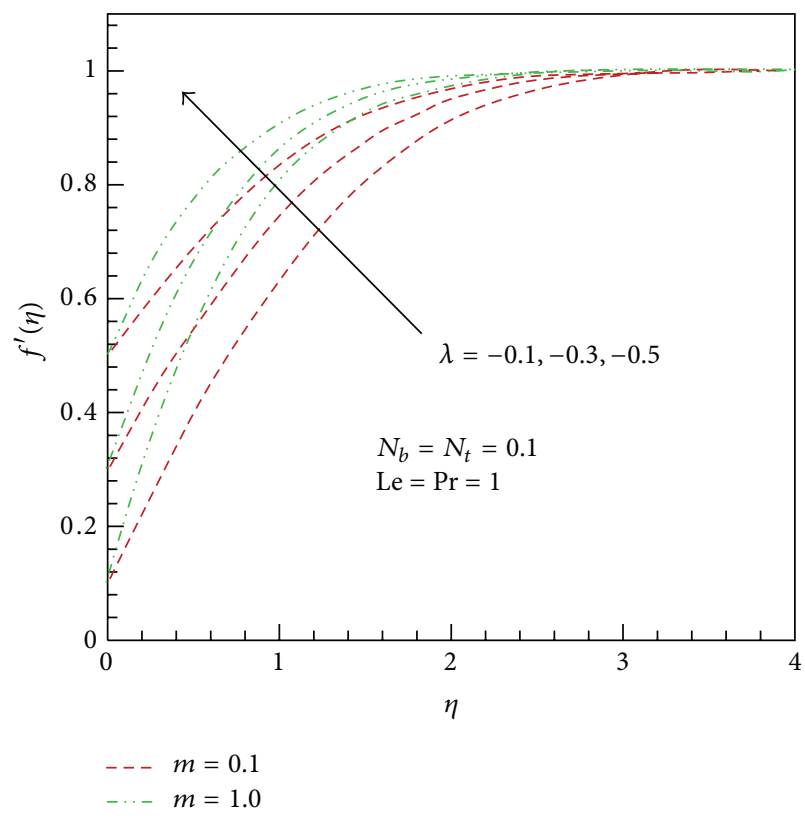

(b)

FIGURE 2: The dimensionless velocity profiles for different values of $m$ along (a) shrinking and (b) stretching wedge.

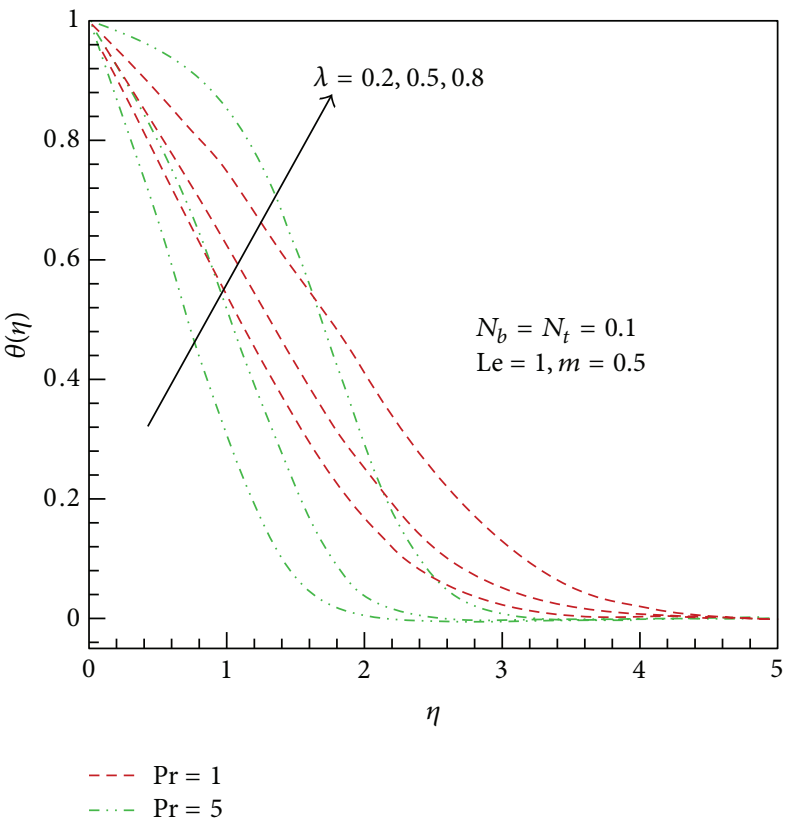

(a)

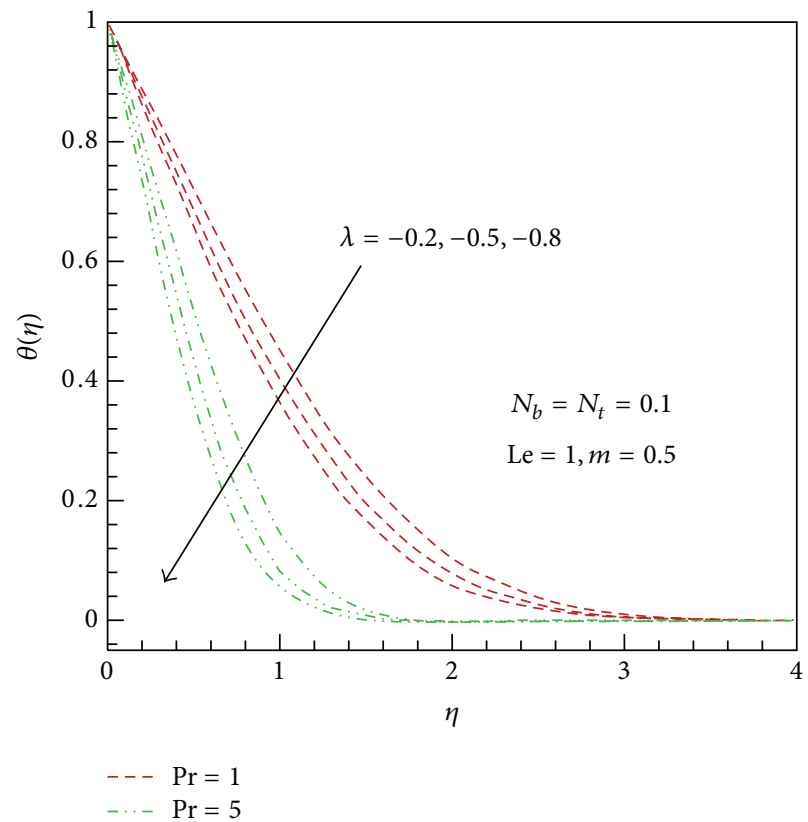

(b)

FIgURE 3: The dimensionless temperature profiles for different values of Pr along (a) shrinking and (b) stretching wedge.

This can be observed in both cases. The effects of nanofluid parameters on the dimensionless temperature are illustrated in Figures 4(a) and 4(b) for two different values of the wedge parameter. Within the thermal boundary layer, the dimensionless temperature increases with both Brownian motion and thermophoresis parameters when the wedge is shrinking. No appreciable effect of nanofluid and wedge parameters on the thermal boundary layer thickness could be found.

Figures 5(a) and 5(b) show the variation of the dimensionless nanoparticle volume fraction with Lewis numbers for different values of wedge parameter when the wedge is fixed. It can be seen that the thickness of concentration boundary layer decreases with increasing Lewis number 


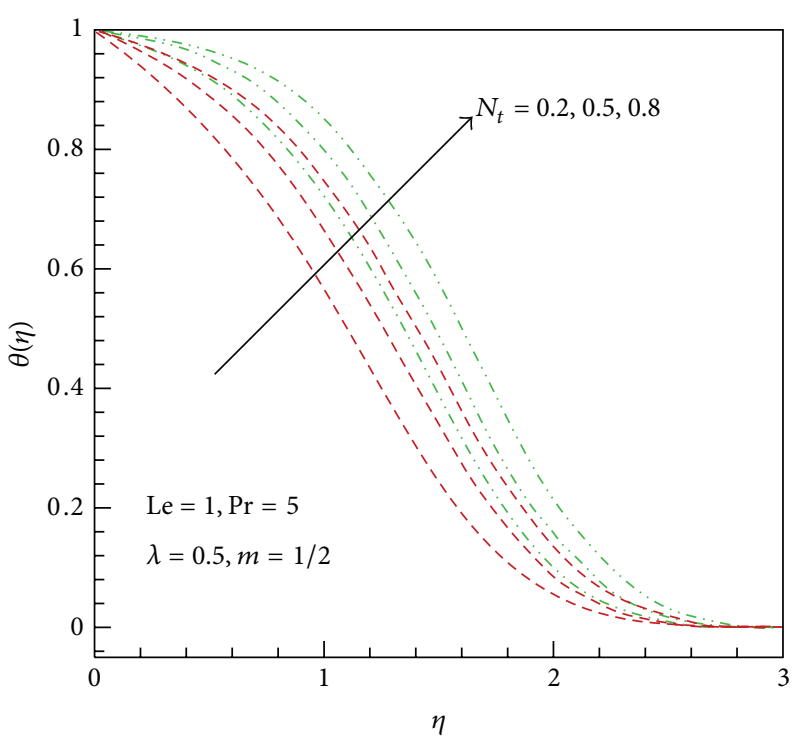

$--N_{b}=0.1$

-.- $N_{b}=0.5$

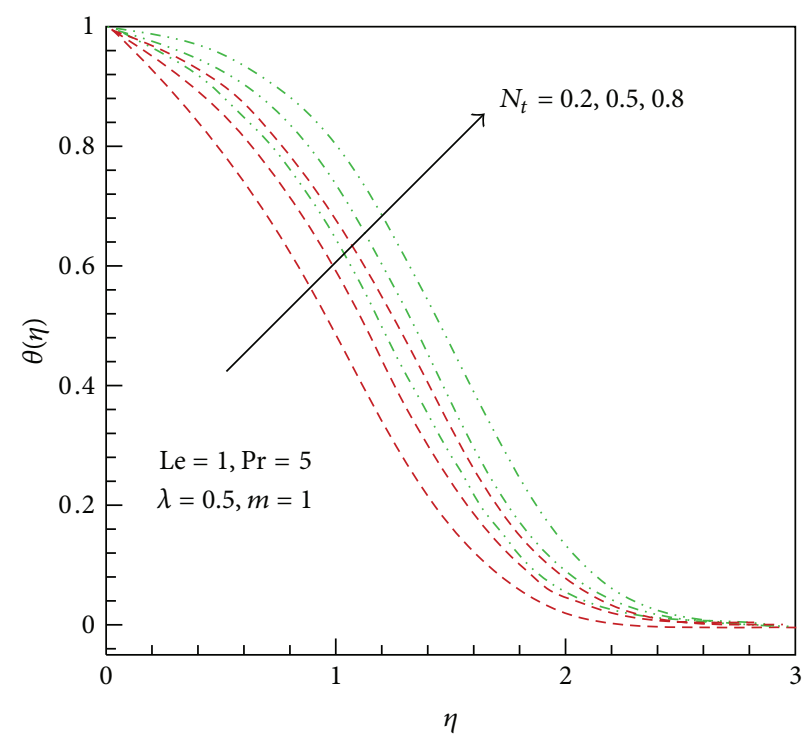

$--N_{b}=0.1$

(a)

(b)

FIGURE 4: Effect of nanofluid parameters on dimensionless temperature along shrinking wedge for (a) $m=1 / 2$ and (b) $m=1$.

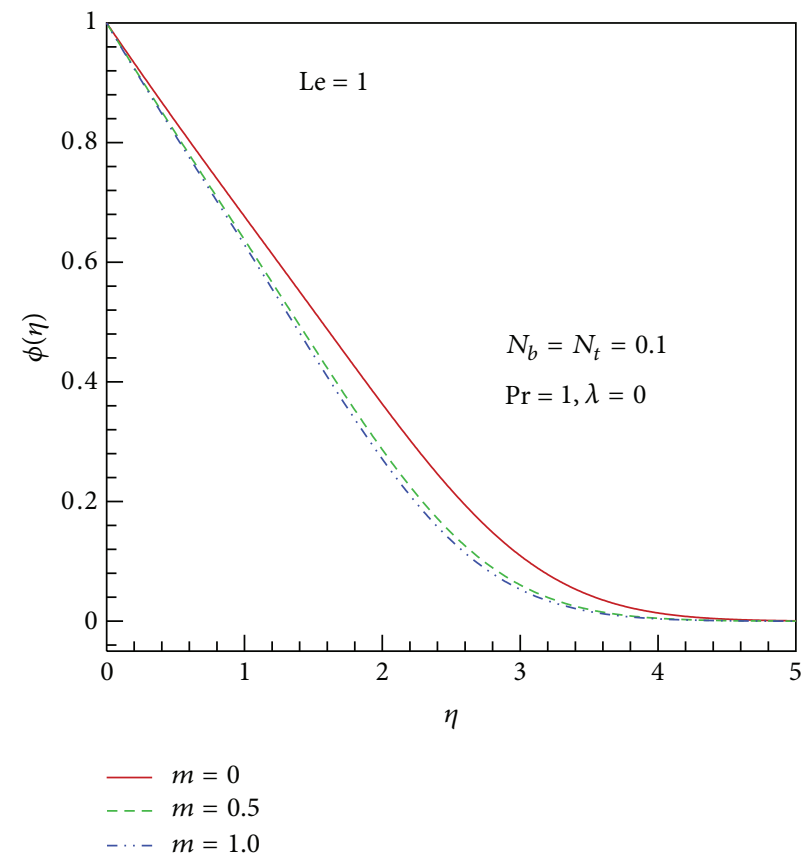

(a)

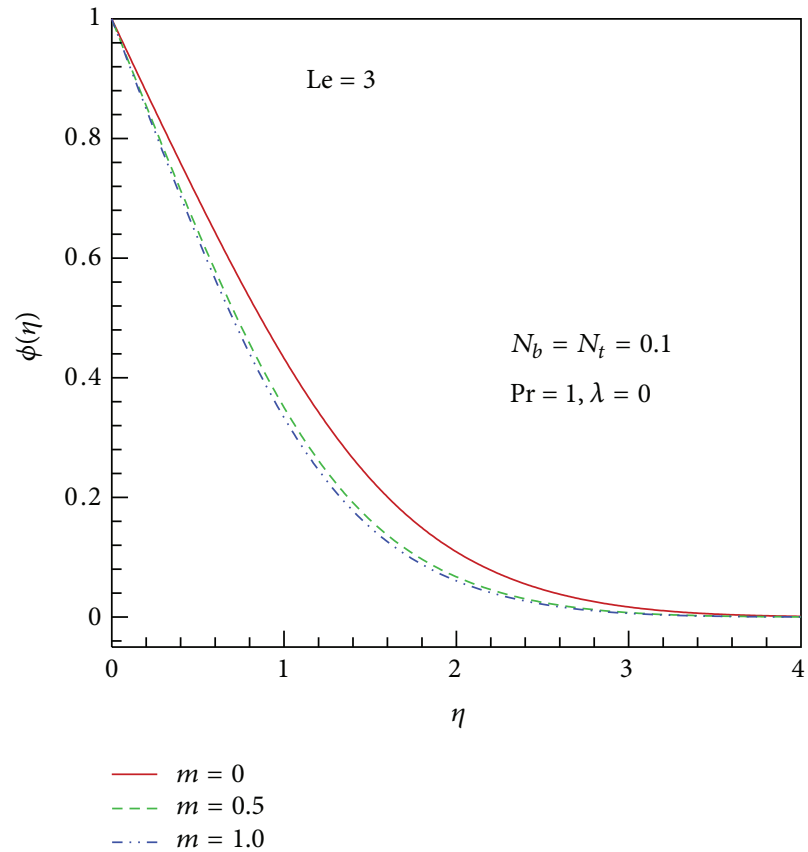

(b)

FIGURE 5: Effects of Lewis numbers on dimensionless nanoparticle volume fraction profiles for different values of $m$ when the wedge is fixed.

and wedge parameter in both cases. Inside the concentration boundary layer, the dimensionless nanoparticle volume fraction is higher for the horizontal flat plate. The effects of nanofluid parameters on dimensionless nanoparticle volume fraction are depicted in Figures 6(a) and 6(b) for shrinking/stretching wedge. In both cases, the concentration boundary layer thickness decreases with increasing Brownian motion and thermophoresis parameters. 


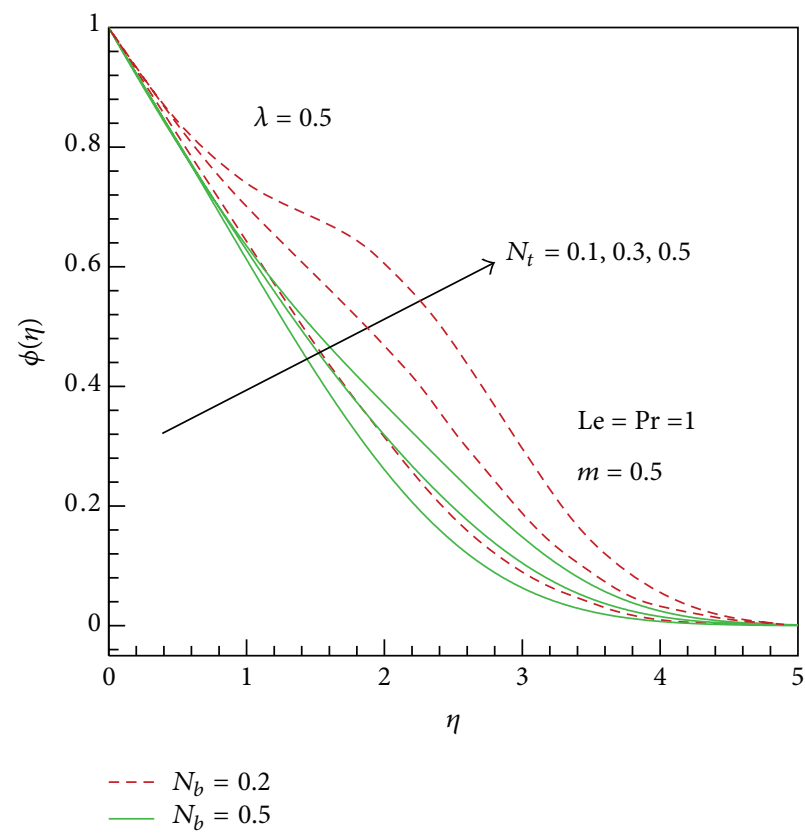

(a)

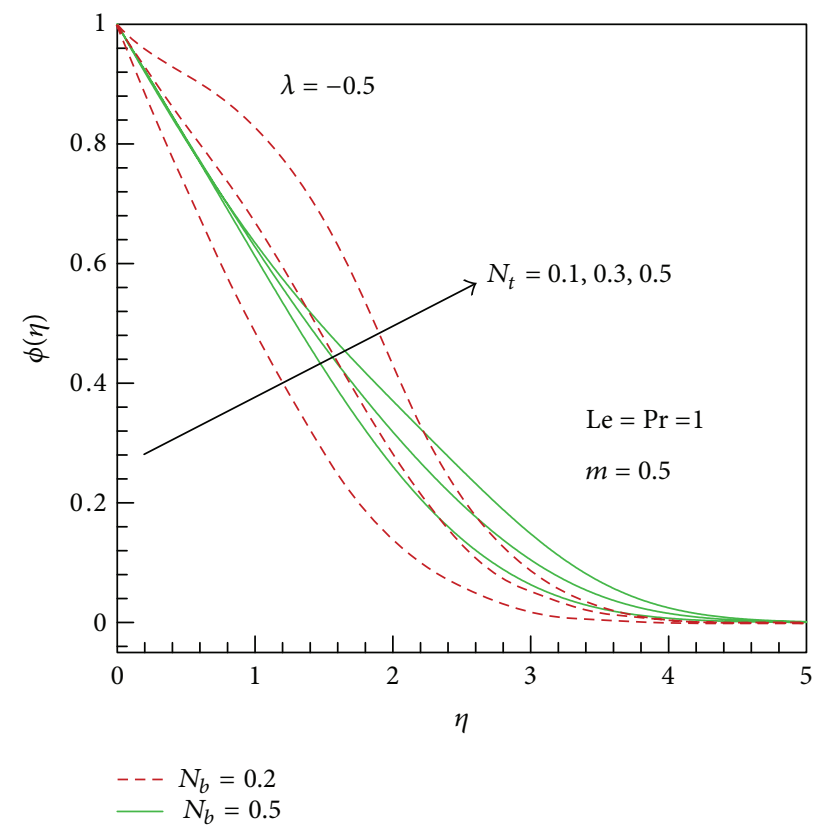

(b)

FIGURE 6: Effects of nanofluid parameters on dimensionless nanoparticle volume fraction for (a) shrinking wedge and (b) stretching wedge.

\section{Conclusions}

Steady boundary layer flow past a moving wedge in a waterbased nanofluid is studied numerically using an implicit finite-difference method for several values of the parameters $m, \lambda, \operatorname{Pr}$, Le, $N_{b}$, and $N_{t}$. This problem reduces to the classical Falkner-Skan's [1] problem of the boundary layer flow of a viscous (Newtonian) fluid past a fixed wedge, when $\lambda, N_{b}$, and $N_{t}$ are all zero. The effects of all these parameters on the dimensionless velocity, temperature, and nanoparticle volume fraction are investigated and presented graphically. It is found that the

(i) dimensionless velocity at the surface increases/ decreases with stretching/shrinking parameters;

(ii) dimensionless temperature increases with both Brownian motion and thermophoresis parameters.

\section{Acknowledgment}

The authors wish to thank the very competent reviewers for their valuable comments and suggestions.

\section{References}

[1] V. M. Falkner and S. W. Skan, "Some approximate solutions of the boundary-layer equations," Philosophical Magazine, vol. 12, pp. 865-896, 1931.

[2] D. R. Hartree, "On an equation occurring in Falkner and Skan's approximate treatment of the equations of the boundary layer," Mathematical Proceedings of the Cambridge Philosophical Society, vol. 33, no. 2, pp. 223-239, 1937.
[3] K. Stewartson, "Further solutions of the Falkner-Skan equation," Mathematical Proceedings of the Cambridge Philosophical Society, vol. 50, pp. 454-465, 1954.

[4] K. K. Chen and P. A. Libby, "Boundary layers with small departure from the Falkner-Skan profile," Journal of Fluid Mechanics, vol. 33, no. 2, pp. 273-282, 1968.

[5] K. R. Rajagopal, A. S. Gupta, and T. Y. Na, "A note on the Falkner-Skan flows of a non-Newtonian fluid," International Journal of Non-Linear Mechanics, vol. 18, no. 4, pp. 313-320, 1983.

[6] E. F. F. Botta, F. J. Hut, and A. E. P. Veldman, "The role of periodic solutions in the Falkner-Skan problem for $\lambda>0$," Journal of Engineering Mathematics, vol. 20, no. 1, pp. 81-93, 1986.

[7] P. Brodie and W. H. H. Banks, "Further properties of the Falkner-Skan equation," Acta Mechanica, vol. 65, no. 1-4, pp. 205-211, 1987.

[8] R. S. Heeg, D. Dijkstra, and P. J. Zandbergen, "The stability of Falkner-Skan flows with several inflection points," Journal of Applied Mathematics and Physics, vol. 50, no. 1, pp. 82-93, 1999.

[9] M. B. Zaturska and W. H. H. Banks, "A new solution branch of the Falkner-Skan equation," Acta Mechanica, vol. 152, no. 1-4, pp. 197-201, 2001.

[10] B. L. Kuo, "Application of the differential transformation method to the solutions of Falkner-Skan wedge flow," Acta Mechanica, vol. 164, no. 3-4, pp. 161-174, 2003.

[11] A. Pantokratoras, "The Falkner-Skan flow with constant wall temperature and variable viscosity," International Journal of Thermal Sciences, vol. 45, no. 4, pp. 378-389, 2006.

[12] S. J. Liao, "A uniformly valid analytic solution of twodimensional viscous flow over a semi-infinite flat plate," Journal of Fluid Mechanics, vol. 385, pp. 101-128, 1999.

[13] S. Abbasbandy and T. Hayat, "Solution of the MHD FalknerSkan flow by homotopy analysis method," Communications in 
Nonlinear Science and Numerical Simulation, vol. 14, no. 9-10, pp. 3591-3598, 2009.

[14] C. S. Liu and J. R. Chang, "The Lie-group shooting method for multiple-solutions of Falkner-Skan equation under suctioninjection conditions," International Journal of Non-Linear Mechanics, vol. 43, no. 9, pp. 844-851, 2008.

[15] T. Fang and J. Zhang, "An exact analytical solution of the Falkner-Skan equation with mass transfer and wall stretching," International Journal of Non-Linear Mechanics, vol. 43, no. 9, pp. 1000-1006, 2008.

[16] D. Pal and H. Mondal, "Influence of temperature-dependent viscosity and thermal radiation on MHD forced convection over a non-isothermal wedge," Applied Mathematics and Computation, vol. 212, no. 1, pp. 194-208, 2009.

[17] S. D. Harris, D. B. Ingham, and I. Pop, "Unsteady heat transfer in impulsive Falkner-Skan flows: constant wall temperature case," European Journal of Mechanics, vol. 21, no. 4, pp. 447-468, 2002.

[18] S. D. Harris, D. B. Ingham, and I. Pop, "Unsteady heat transfer in impulsive Falkner-Skan flows: constant wall heat flux case," Acta Mechanica, vol. 201, no. 1-4, pp. 185-196, 2008.

[19] S. D. Harris, D. B. Ingham, and I. Pop, "Impulsive FalknerSkan flow with constant wall heat flux: revisited," International Journal of Numerical Methods for Heat and Fluid Flow, vol. 19, no. 8, pp. 1008-1037, 2009.

[20] W. H. H. Banks, "Similarity solutions of the boundary-layer equations for a stretching wall," Journal of Theoretical and Applied Mechanics, vol. 2, no. 3, pp. 375-392, 1983.

[21] J. Serrin, "Asymptotic behavior of velocity profiles in the Prandtl boundary layer theory," Proceedings of the Royal Society A, vol. 299, pp. 491-507, 1967.

[22] N. Riley and P. D. Weidman, "Multiple solutions of the FalknerSkan equation for flow past a stretching boundary," SIAM Journal on Applied Mathematics, vol. 49, no. 5, pp. 1350-1358, 1989.

[23] A. Ishak, R. Nazar, and I. Pop, "Falkner-Skan equation for flow past a moving wedge with suction or injection," Journal of Applied Mathematics \& Computing, vol. 25, no. 1-2, pp. 67-83, 2007.

[24] Y. Xuan and W. Roetzel, "Conceptions for heat transfer correlation of nanofluids," International Journal of Heat and Mass Transfer, vol. 43, no. 19, pp. 3701-3707, 2000.

[25] A. V. Kuznetsov and D. A. Nield, "Boundary layer treatment of forced convection over a wedge with an attached porous substrate," Journal of Porous Media, vol. 9, no. 7, pp. 683-694, 2006.

[26] D. A. Nield and A. V. Kuznetsov, "The Cheng-Minkowycz problem for natural convective boundary-layer flow in a porous medium saturated by a nanofluid," International Journal of Heat and Mass Transfer, vol. 52, no. 25-26, pp. 5792-5795, 2009.

[27] A. V. Kuznetsov and D. A. Nield, "Double-diffusive natural convective boundary-layer flow of a nanofluid past a vertical plate," International Journal of Thermal Sciences, vol. 50, no. 5, pp. 712-717, 2011

[28] D. A. Nield and A. V. Kuznetsov, "The Cheng-Minkowycz problem for the double-diffusive natural convective boundary layer flow in a porous medium saturated by a nanofluid," International Journal of Heat and Mass Transfer, vol. 54, no. 1-3, pp. 374-378, 2011.

[29] P. Cheng and W. J. Minkowycz, "Free convection about a vertical flat plate embedded in a porous medium with application too heat transfer from a dike," Journal of Geophysical Research, vol. 82, no. 14, pp. 2040-2044, 1977.
[30] A. V. Kuznetsov and D. A. Nield, "Natural convective boundarylayer flow of a nanofluid past a vertical plate," International Journal of Thermal Sciences, vol. 49, no. 2, pp. 243-247, 2010.

[31] M. Narayana and P. Sibanda, "Laminar flow of a nanoliquid film over an unsteady stretching sheet," International Journal of Heat and Mass Transfer, vol. 55, no. 25-26, pp. 7552-7560, 2012.

[32] P. K. Kameswaran, M. Narayana, P. Sibanda, and P. V. S. N. Murthy, "Hydromagnetic nanofluid flow due to a stretching or shrinking sheet with viscous dissipation and chemical reaction effects," International Journal of Heat and Mass Transfer, vol. 55, no. 25-26, pp. 7587-7595, 2012.

[33] P. K. Kameswaran, S. Shaw, P. Sibanda, and P. V. S. N. Murthy, "Homogeneous-heterogeneous reactions in a nanofluid flow due to a porous stretching sheet," International Journal of Heat and Mass Transfer, vol. 57, no. 2, pp. 465-472, 2013.

[34] K. F. V. Woong and O. D. Leon, "Applications of nanofluids: current and future," Advances in Mechanical Engineering, vol. 2010, Article ID 519659, 11 pages, 2010.

[35] R. Saidur, S. N. Kazi, M. S. Hossain, M. M. Rahman, and H. A. Mohamed, "A review on the performance of nanoparticles suspended with refrigerants and lubricating oils in refrigeration systems," Renewable and Sustainable Energy Reviews, vol. 15, no. 1, pp. 310-323, 2011.

[36] O. Mahian, A. Kianifar, S. A. Kalogirou, I. Pop, and S. Wongwises, "A review of the applications of nanofluids in solar nnergy," International Journal of Heat and Mass Transfer, vol. 57, no. 2, pp. 582-594, 2013.

[37] O. Behar, A. Khellaf, and K. Mohammedi, "A review of studies on central receiver solar thermal power plants," Renewable and Sustainable Energy Reviews, vol. 23, pp. 12-39, 2013.

[38] K. A. Yih, "Uniform suction/blowing effect on forced convection about a wedge: uniform heat flux," Acta Mechanica, vol. 128, no. 3-4, pp. 173-181, 1998.

[39] N. A. Yacob, A. Ishak, and I. Pop, "Falkner-Skan problem for a static or moving wedge in nanofluids," International Journal of Thermal Sciences, vol. 50, no. 2, pp. 133-139, 2011.

[40] F. M. White, Viscous Fluid Flow, McGraw-Hill, New York, NY, USA, 2nd edition, 1991.

[41] B. L. Kuo, "Heat transfer analysis for the Falkner-Skan wedge flow by the differential transformation method," International Journal of Heat and Mass Transfer, vol. 48, no. 23-24, pp. 50365046, 2005.

[42] H. Blasius, "Grenzschichten in Flüssigkeiten mit kleiner Reibung," Zentralblatt Mathematical Physics, vol. 56, pp. 1-37, 1908.

[43] T. Cebeci and P. Bradshaw, Momentum Transfer in Boundary Layers, Hemisphere Publishing Corporation, New York, NY, USA, 1977.

[44] T. Cebeci and P. Bradshaw, Physical and Computational Aspects of Convective Heat Transfer, Springer, New York, NY, USA, 1988. 


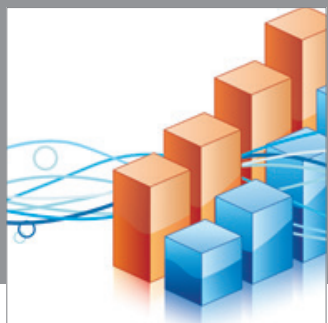

Advances in

Operations Research

mansans

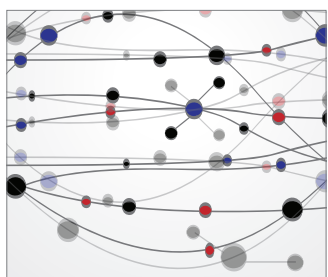

The Scientific World Journal
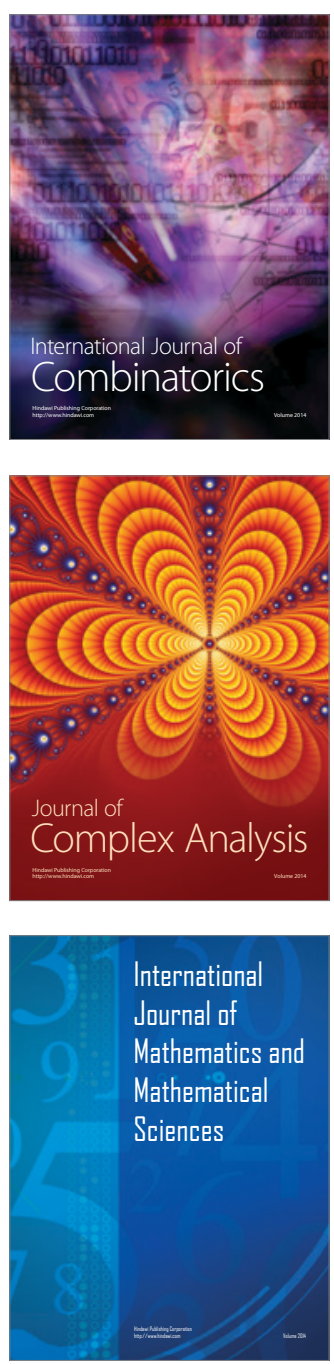
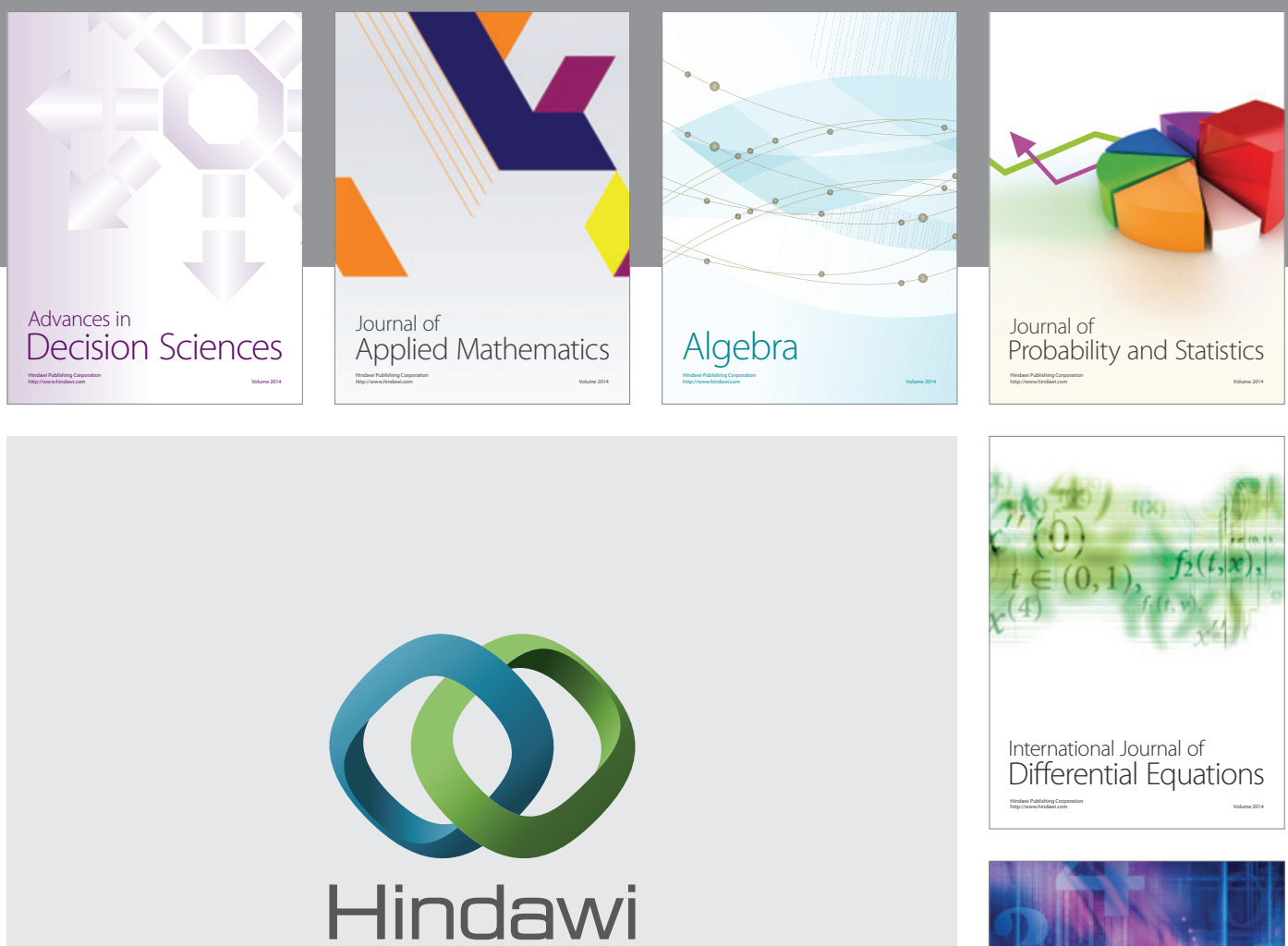

Submit your manuscripts at http://www.hindawi.com
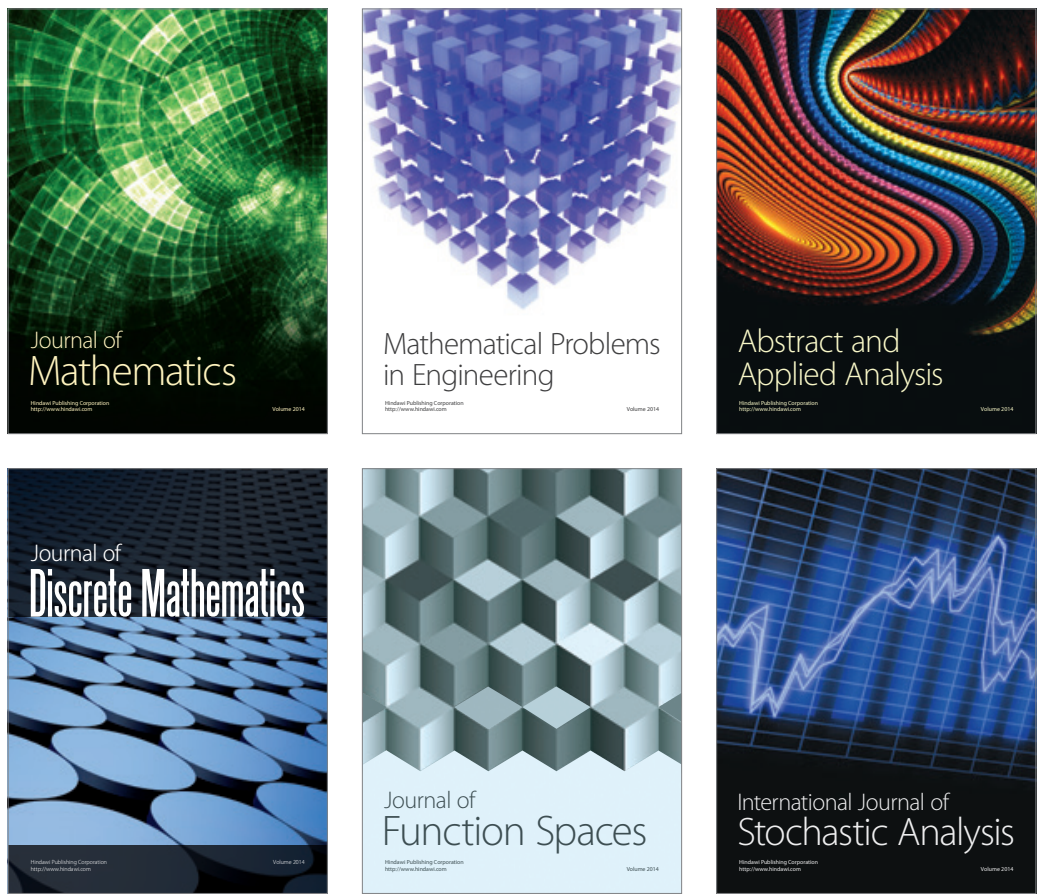

Journal of

Function Spaces

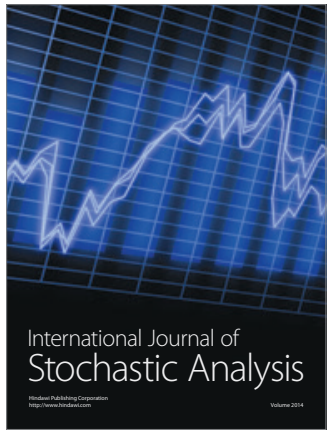

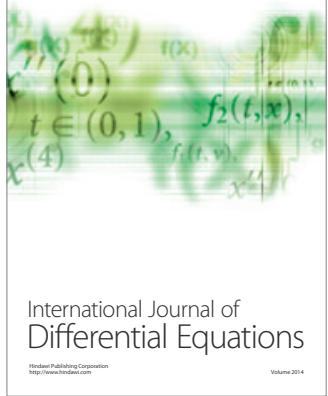
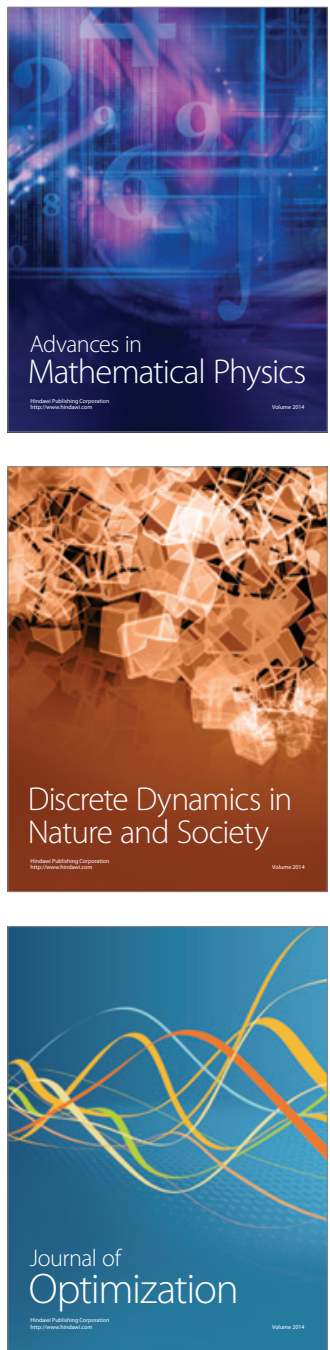